\title{
QUEEN'S
UNIVERSITY
BELFAST
}

\section{Local probing of ferroelectric and ferroelastic switching through stress- mediated piezoelectric spectroscopy}

Edwards, D., Brewer, S., Cao, Y., Jesse, S., Chen, L-Q., Kalinin, S. V., Kumar, A., \& Bassiri-Gharb, N. (2016).

Local probing of ferroelectric and ferroelastic switching through stress-mediated piezoelectric spectroscopy.

Advanced Materials Interfaces, 3(7). https://doi.org/10.1002/admi.201500470

Published in:

Advanced Materials Interfaces

Queen's University Belfast - Research Portal:

Link to publication record in Queen's University Belfast Research Portal

\section{Publisher rights}

(c) 2016 WILEY-VCH Verlag GmbH \& Co. KGaA, Weinheim

This is the peer reviewed version of the following article: Edwards D., Brewer S., Cao Y., Jesse S., Chen Long-Qing, Kalinin S. V., Kumar A., Bassiri-Gharb N. (2016). Local Probing of Ferroelectric and Ferroelastic Switching through Stress-Mediated Piezoelectric Spectroscopy. Adv. Mater. Interfaces, 3: . doi: 10.1002/admi.201500470, which has been published in final form at http://dx.doi.org/10.1002/admi.201500470.

This article may be used for non-commercial purposes in accordance with Wiley Terms and Conditions for Self-Archiving."

\section{General rights}

Copyright for the publications made accessible via the Queen's University Belfast Research Portal is retained by the author(s) and / or other copyright owners and it is a condition of accessing these publications that users recognise and abide by the legal requirements associated with these rights.

Take down policy

The Research Portal is Queen's institutional repository that provides access to Queen's research output. Every effort has been made to ensure that content in the Research Portal does not infringe any person's rights, or applicable UK laws. If you discover content in the

Research Portal that you believe breaches copyright or violates any law, please contact openaccess@qub.ac.uk. 


\section{WILEY-VCH}

DOI: 10.1002/ ((please add manuscript number))

Article type: Full Paper

\section{Local probing of ferroelectric and ferroelastic switching through stress-mediated piezoelectric spectroscopy}

David Edwards, Steven Brewer, Ye Cao, Stephen Jesse, Long-Qing Chen, Sergei V. Kalinin, Amit Kumar* and Nazanin Bassiri-Gharb*

D. Edwards, Dr. A. Kumar

School of Mathematics and Physics, Queen's University Belfast, Belfast, BT7 1NN, United

Kingdom

E-mail: a.kumar@qub.ac.uk

S. Brewer, Prof. N. Bassiri-Gharb

G.W. Woodruff School of Mechanical Engineering, Georgia Institute of Technology, Atlanta, Georgia, 30332-0405, USA

E-mail: nazanin.bassiri@gatech.edu

Dr. Y. Cao, Dr. S. Jesse, Dr. S. V. Kalinin

Center for Nanophase Materials Sciences, Oak Ridge National Laboratory, Oak Ridge,

Tennessee, 37831, USA

Prof. L. Q. Chen

Department of Materials Science and Engineering, The Pennsylvania State University, University Park, Pennsylvania, 16802, USA

Keywords: ferroelectric, ferroelastic, piezoresponse force microscopy, lead zirconate titanate

Strain effects have a significant role in mediating classic ferroelectric behavior such as polarization switching and domain wall dynamics. These effects are of critical relevance if the ferroelectric order parameter is coupled to strain and is therefore, also ferroelastic. Here, we combine switching spectroscopy piezoresponse force microscopy (SS-PFM) with control of applied tip pressure, to exert direct control over the ferroelastic and ferroelectric switching events, a modality otherwise unattainable in traditional PFM. As a proof of concept, we have applied stress-mediated SS-PFM towards the study of polarization switching events in a lead zirconate titanate thin film, with a composition near the morphotropic phase boundary with co-existing rhombohedral and tetragonal phases. Under increasing applied pressure, we observe shape modification of local hysteresis loops, consistent with a reduction in the 


\section{WILEY-VCH}

ferroelastic domain variants under increased pressure. These experimental results are further validated by phase field simulations. The technique can be expanded to explore more complex electromechanical responses under applied local pressure, such as probing ferroelectric and ferroelastic piezoelectric nonlinearity as a function of applied pressure, and electro-chemomechanical response through electrochemical strain microscopy (ESM).

\section{Introduction}

Switchable spontaneous polarization under an applied electric field is the characteristic feature of ferroelectric materials, with clear relevance for memory applications. $^{[1,2]}$ Additionally, while all ferroelectric materials are also pyroelectric and piezoelectric, many ferroelectrics are also ferroelastic, i.e. the order parameter is coupled to strain. The resulting enhancement of the electromechanical coupling is key for application of these materials in sensors and actuators, both in bulk applications and for microelectromechanical systems (MEMS). ${ }^{[3]}$ The role of strain in mediating classical ferroelectric behavior, such as polarization switching ${ }^{[4]}$ and domain wall dynamics, ${ }^{[5]}$ is an equally important aspect of electromechanical coupling in such materials. The importance of strain/stress boundary conditions with respect to the functional properties of ferroelectric materials is especially relevant at the micro- and nano-scale for the end technological applications. For example, mechanical clamping in polycrystalline thin films can reduce the

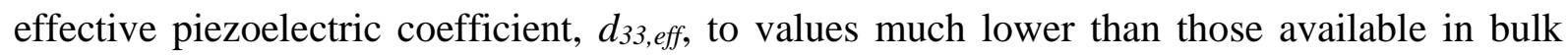
ceramics. ${ }^{[6]}$ Epitaxial strain can be engineered to result in enhancement of ferroelectric ${ }^{[7]}$ and piezoelectric $^{[4]}$ response, induce ferroelectric behavior in materials that are otherwise not ferroelectric, ${ }^{[8]}$ or alternatively suppress the ferroelectric transition to much lower temperatures ${ }^{[9]}$ and lead to creation of paraelectric phases. ${ }^{[10]}$ However, stress and strain fields are inherently intertwined with the fundamental ferroelectric characteristics, ${ }^{[11]}$ from creation of the spontaneous strain associated with spontaneous polarization development at transition 


\section{WILEY-VCH}

temperature, to boundary conditions at grain boundaries leading to formation of dense domain structures. ${ }^{[12]}$ These internal interfaces in addition to the eventual phase boundaries ${ }^{[13]}$ are major contributors to the very large electromechanical response of ferroelectric materials (up to $40-80 \%$ in lead zirconate titanate (PZT) ceramics). ${ }^{[14]}$ However, the stress fields associated with each domain wall can result in complex interactions with local defects -be these point defects, dislocations, other domain walls or grain boundaries-, limiting or enhancing the mobility of the domain wall itself. ${ }^{[15,16]}$ In fact, recent studies have illustrated the unexpectedly large role of clamping on the electromechanical response of PZT thin films, suggesting the presence of nontrivial, large length-scales for strain interactions present in such samples. ${ }^{[17]}$ Similarly, evidence has also been provided of avalanche-like behavior of multiple domain walls due to cooperative interactions across these large length scales in disordered ferroelectric thin films. ${ }^{[18]}$

These considerations suggest that the role of local strains on ferroelectric and ferroelastic properties is central to both the piezoelectric and ferroelectric phenomena themselves. In the last two decades, significant progress has been achieved in probing electric field-controlled functionality of ferroelectrics by piezoresponse force microscopy (PFM). In PFM, the localized electric field created by a scanning probe microscopy (SPM) tip induces a local piezoelectric response that can be further related to domain nucleation and domain wall motion processes ${ }^{[19,20,21]}$ providing a very detailed picture of local functionalities. However, the lack of control on mechanical fields results in mostly incomplete information with respect to the separation of the ferroelectric and ferroelastic interfaces, unless complicated threedimensional reconstruction of the domain structure is exploited. ${ }^{[22,23]}$ Therefore, it is imperative to create an approach for local studies of strain-induced phenomena, where external pressure exerted by an SPM probe will directly affect ferroelastic properties of the material during ferroelectric switching. 


\section{WILEY-VCH}

In fact, local mechanical properties have been previously probed via nano-indentation measurements in ferroelectric films where increasing applied pressure has demonstrated mechanically induced cracking, dislocation generation, as well as irreversible domain wall displacements. ${ }^{[24,25,26]}$ Recently, PFM has been used to mechanically write ferroelectric domains in ultrathin barium titanate films via application of localized tip pressure during scanning. ${ }^{[27]}$ In this work, $180^{\circ}$ switching of ferroelectric domains was confirmed with polarization switching deemed to be mediated by flexoelectricity. These results point towards the feasibility of developing an approach where control of applied tip pressure could be used to exert direct control over the ferroelastic and ferroelectric domain switching behavior during electric field induced electromechanical response. In the present work, we capture the effect of applied pressure from a PFM tip on a lead zirconate titanate (PZT) film, while measuring the electric field-induced piezoresponse in a spatially and dynamically resolved manner, i.e. in a combined force-voltage spectroscopy. In these measurements, the SPM probe is a source of local stress field, as well as localized electric field (like in classical PFM), resulting in ferroelastic and ferroelectric switching as discussed below. Furthermore, we investigate the effect of a local stress field on the domain structure of the thin film through phase field simulations.

\section{Results}

As a model system, a $\sim 0.66 \mu \mathrm{m}$ thick $\mathrm{Pb}\left(\mathrm{Zr}_{1-\mathrm{x}} \mathrm{Ti}_{\mathrm{x}}\right) \mathrm{O}_{3}$ film, with a composition $(\mathrm{x} \sim 0.47)$ near the morphotropic phase boundary (MPB) with co-existing rhombohedral and tetragonal phases was chosen for these studies. Piezoresponse imaging at increasing tip force was used to evaluate rearrangement of the domain structure in the film upon application of pressure as shown in Figure 1. The initial topography image in Figure 1a highlights the polycrystalline nature of the film, which consists of (100) textured grains (Supplementary Information Figure S.1) ${ }^{[28]}$ Assuming that an increase in the out-of-plane polarization component of the domains 


\section{WILEY-VCH}

makes the film more amenable to subsequent ferroelastic switching under applied normal load, a region was written with an electric bias of $-6 \mathrm{~V}$, applied from the tip. Vertical PFM amplitude and phase images (Figures $1 \mathrm{~b}$ and 1d) indicate clear polarization switching in the electrically-written region.

In order to apply localized stress to the film, a smaller region within the bias written area was re-written while applying a $5 \mathrm{~V}$ deflection setpoint (indicative of the tip pressure) through the PFM tip, but without applying any DC electric bias. Through the process outlined in the experimental section, we estimate that this setpoint corresponds to an applied force of $1000 \mathrm{nN}$. Assuming the tip to represent a disk with a radius of $20 \mathrm{~nm}$, the applied tip pressure is further estimated to be around $0.8 \mathrm{GPa} .{ }^{[27,29]} \mathrm{A}$ subsequent PFM image (Figure 1c and 1e) clearly reveals a reorganization of the domain structure due to the applied pressure, with a number of domains having a reduced out-of-plane polarization component. Several subsequent scans of the same region revealed that the mechanically written domains were robust even when imaged with a grounded tip under normal loading force, excluding the possibility of the grounded tip causing the reorganization of the domain structure. ${ }^{[30]}$ These results suggest a domain rearrangement mechanism, involving interplay between ferroelectric and ferroelastic switching, which could be induced by either electric field, localized stress or a combination of the two stimuli. ${ }^{[31]}$

In order to systematically probe and isolate the role of applied stress during switching, an adaptation of the switching spectroscopy piezoresponse force microscopy (SS-PFM) technique with direct control over the applied tip force was used. This was done to directly evaluate stress-mediated domain switching while studying electric field-induced electromechanical response within a single set of automated measurements. SS-PFM allows acquisition of the hysteretic electromechanical response of the material through a step-wise DC waveform applied to the tip, over a dense two-dimensional grid of points where a band excitation (BE) signal is used to excite and detect the response at each DC step (BE- 


\section{WILEY-VCH}

SSPFM).${ }^{[32]}$ In contrast to the single frequency signal used in standard PFM techniques, a BE signal consists of a waveform with a defined amplitude and phase over a range of frequencies in proximity to the tip-surface contact resonance. ${ }^{[33]}$ In the adapted technique, the loading force applied thorough the tip adds a further dimension to the hysteretic behavior studied using SSPFM in a spatially resolved manner (Figure S2). This approach was used to acquire evolution of the hysteretic response on the surface of the PZT film (without a top electrode) with increasing and decreasing tip force, as displayed in Figure 2. The behavior shown in Figure $2 \mathrm{a}$ and $2 \mathrm{~b}$ is a typical shape obtained at most locations on the surface of the film, albeit minor variations are also observed across the surface. At lower tip forces, the piezoresponse loops show a typical hysteresis shape with simple saturation observed at both increasing and decreasing bias. In contrast, the hysteresis curves at higher tip forces show loops with "noses", i.e. the doubling up of the hysteresis curve on itself and a change in the travel direction from counter clockwise to locally clockwise at negative bias values. This highlights a different switching behavior under an external applied electric field at higher levels of applied pressure. As the loading force is decreased (Figure 2b) the switching behavior appears to revert back to a more typical hysteresis shape (albeit with a slight increase in coercive field) indicating an extent of reversibility in the role of applied pressure on the bias induced switching of the film.

An advantage of utilizing a BE signal to excite and probe the piezoresponse of the film is that it mostly overcomes topographic crosstalk problems associated with the spatial variations of the contact resonance frequency. ${ }^{[34]}$ Additionally, it allows the extraction of the tip-surface contact resonance frequency as a function of varying tip bias and force as shown in Figure 3. From this data, it is clear that an increased applied tip force is accompanied with an increase of the tip-surface contact resonance. As will be discussed later, this increase can be attributed to a hardening of the material with increased applied pressure. The contact resonance decreases back to initial values upon decreasing the loading force. In these 


\section{WILEY-VCH}

experiments, the measurements were performed in absence of a top electrode, locally probing the effect of applied tip pressure on the electromechanical response induced by a radially decaying electric field. A further point of interest is to investigate the stress-mediated hysteretic behavior when the applied electric field is uniform. This scenario was realized through the deposition of a macroscopic Pt top electrode on the film and the experiment was repeated on the electrode. In this case, little to no variation in the piezoresponse hysteresis loop shape or area as a function of the applied tip force was observed (Figure 4). Thus, the uniformity of the electric field applied via the tip significantly influences the switching behavior.

In order to further rationalize the obtained experimental results and investigate the effect of tip pressure directly on the ferroelectric-ferroelastic domain structure, the mechanical switching dynamics of PZT films were modeled by a phase field simulation. ${ }^{[35,36]} \mathrm{A} \mathrm{Pt}$ indenter tip of radius $20 \mathrm{~nm}$, modeled by a spherical indenter model, ${ }^{[37]}$ applies $600 \mathrm{nN}$ of force (or $\sim 0.5 \mathrm{GPa}$ of pressure) to $50 \mathrm{~nm}$ thick $\mathrm{Pb}\left(\mathrm{Zr}_{1-\mathrm{x}} \mathrm{Ti}_{\mathrm{x}}\right) \mathrm{O}_{3}$ thin films of various compositions $(x=0.2,0.47$ and 0.8 ) (results for $\mathrm{x}=0.8$ and $\mathrm{x}=0.2$ are displayed in Figures S3 and S4, respectively). The initial domain structure generated by the simulation at the MPB composition $(x=0.47)$ is shown in Figure 5a. The tip is then applied to four different rhombohedral domain variants at the surface. The mechanical pressure is seen to induce rhombohedral-rhombohedral (R-R) and rhombohedral-tetragonal (R-T) domain switching, while orthorhombic domains are also observable at R-R and R-T domain boundaries. In contrast to the results for $\mathrm{x}=0.8$ and 0.2 (see Supplementary Information), domain switching appears to penetrate the entire film thickness, highlighting that the MPB composition is indeed softer than either purely tetragonal or purely rhombohedral compositions.

\section{Discussion}




\section{WILEY-VCH}

Mechanical writing of electrically-written domains has been previously reported for very thin $\left(<5 \mathrm{~nm}\right.$ thick) films of $\mathrm{BaTiO}_{3}$, where ferroelectric switching, mediated through flexoelectricity, was proposed as the dominant mechanism. ${ }^{[27]}$ However, the films probed in our work are substantially thicker: the field gradients exerted by the tip within the sample are expected to permeate a much smaller fraction of the hundreds of nanometers thick films, and hence the switching we observed cannot be uniquely explained by the flexoelectric mechanism. Other potential mechanisms, such as piezochemical effect, ${ }^{[38]}$ have also been used to explain mechanical writing in thin films of oxides but the films used in such studies were also quite thin $(50 \mathrm{~nm})$. In fact, chemical coupling with electromechanical response usually requires film thicknesses comparable to the diffusion length scale for the mobile chemical species, ${ }^{[38]}$ which is indeed substantially smaller than the $660 \mathrm{~nm}$ thick PZT films under discussion. The most likely mechanism for mechanical writing in these films is ferroelastic domain wall movement caused by external mechanical forces applied via the tip. The mechanical loading of the tip is expected to move the ferroelastically-active domain walls, resulting in rearrangement of the domain structure as observed in both the piezoresponse image (Figure 1) and the phase field simulation (Figure 5). The dynamic studies meanwhile reveal unique behavior in the PZT films with coexisting rhombohedral and tetragonal domains. The piezoresponse hysteresis curves observed at low applied pressures are those typically reported in ferroelastic materials. Conversely, the nose and hump observed in the loops at high tip loading have been previously reported to be associated with purely ferroelectric, $180^{\circ}$ domain wall switching contributions. ${ }^{[39]}$

In a more detailed discussion of the mechanisms, we first consider the possible ferroelastic variants in this material system. The (100) orientation of the film does not allow any preferential polarization variants with respect to the out-of-plane direction for the rhombohedral composition: all polarization directions have the same absolute out-of-plane and in-plane components. Conversely, in the tetragonal phase, presence of " $a$ " (fully in-plane 


\section{WILEY-VCH}

polarization direction) and " $c$ " (fully out-of-plane polarization direction) domain variants leads to presence of ferroelastically active domain walls. Last but not least, any eventual transitions between the rhombohedral and tetragonal phase, and vice versa, would result in variations of the in-plane and out-of-plane polarization components and therefore would be also ferroelastically active. As the pressure under the tip increases, the polarization in the film can be expected to switch in such way to reduce the out-of-plane component, while increasing the in-plane component. With respect to the ferroelastic variants discussed above, there are therefore three possible ways in which polarization reorientation can be achieved in these samples, which are discussed below and schematically illustrated in Figure 6.

In-plane polarization components might be available due to slight misorientation of the (100) texturing of the rhombohedral grains. As mentioned earlier, in the ideal (100) textured rhombohedral grains, all the polarization directions are energetically degenerate (in absolute value) with respect to their out-of-plane component: therefore, application of the normal load should not effectively modify the domain state. A less-than perfect alignment of the crystal will however remove the ideal degenerate state of the eight polarization directions with respect to the normal to the plane of the film, resulting in some polarization directions with slightly larger in-plane components with respect to others (and slightly smaller out-ofplane components). However, such departure from degeneracy is not expected to make a large contribution to the electromechanical response, considering the limited volume of the sample with non-(100) texturing. Based on XRD data reported elsewhere, less than $\sim 3 \%$ departure from (100) orientation is expected in the studied films. ${ }^{[28]}$

A more probable alternative involves a phase transition under mechanical load, based on the proximity to the MPB. Specifically, a transition from rhombohedral to tetragonal phase " $a$ " domain $\left(\mathrm{R} \rightarrow \mathrm{T}_{\mathrm{a}}\right)$, such as that seen in the phase field simulations (Figure 5e), can be expected under the applied normal load. Such a transition would result in an increase in the in-plane polarization component. Similarly, within the tetragonal phase, at increasing normal 


\section{WILEY-VCH}

load, switching from " $c$ " to " $a$ " domains can be expected. This is also consistent with the phase field simulation for $\mathrm{x}=0.8$, where " $c$ " to " $a$ " domain switching events $\left(\mathrm{T}_{\mathrm{c}} \rightarrow \mathrm{T}_{\mathrm{a}}\right)$ are visible near the surface of the film (Figure S3b). However such "c" to " $a$ " domain switching events are expected to be more limited, as processing of PZT thin films on platinized Si wafers $^{[40]}$ usually results in ferroelectric films under residual tensile stress, leading to a large portion of the domains variants with in-plane-polarization components (as allowed by crystallographic structure and orientation). ${ }^{[11]}$ Nonetheless, at increasing thickness of the ferroelectric film, the residual stress decays logarithmically ${ }^{[42]}$ and therefore, in the immediate proximity of the ferroelectric film surface, both " $a$ " and " $c$ " domains could be available. ${ }^{[43]}$

At low tip pressures, local ferroelastic domain switching via the above-described $\mathrm{R} \rightarrow$ $\mathrm{T}_{\mathrm{a}}$ and $\mathrm{T}_{\mathrm{c}} \rightarrow \mathrm{T}_{\mathrm{a}}$ mechanisms should be also achievable through applied electric field at low tip pressures. Such ferroelastic switching will therefore dominate the overall piezoresponse of the sample (Figure 2a and 2b). At higher tip pressures, however, the ferroelastic variants are mostly exhausted through the same $\mathrm{R} \rightarrow \mathrm{T}_{\mathrm{a}}$ and $\mathrm{T}_{\mathrm{c}} \rightarrow \mathrm{T}_{\mathrm{a}}$ switching mechanisms, due to the stress fields created. Therefore, the electromechanical response probed through application of electric field is increasingly dominated by contributions from ferroelectric, $180^{\circ}$ domain wall motions. ${ }^{[39,44,45]}$ The change in the electromechanical response under increasing applied pressure is also comparable with previous studies carried out on thinner $(<300 \mathrm{~nm})$ PZT films of similar composition $(\mathrm{Zr}: \mathrm{Ti}=52: 48) .{ }^{[46]}$ However, in the work by Zavala et al., the quantitative reduction in the effective piezoelectric coefficient as a function of the applied load was measured at a single frequency, and therefore could be affected by calibration artifacts due to the reduced motion of the full cantilever, and not necessarily that of the sample. However, the use of band excitation precludes large artifacts induced by the clamping motion of the cantilever at larger set points, and observed variations in the piezoresponse are mostly due to the material's response and reduction/exhaustion of ferroelastic contributions. 


\section{WILEY-VCH}

While at substantially different load levels and length scales, our finding is also partially consistent with previous reports by Krueger ${ }^{[47]}$ in bulk PZT ceramics for compressive stress parallel to the polar axis: an increasing load can result in substantial changes in the polarization state of the ferroelectric, resulting in an initial increase in the electromechanical response, followed by a decrease at removal of the load. In hard PZT ceramics, specifically, the degradation under load is mostly recovered at unloading. For soft PZT ceramics, however, the hardening is not recoverable. The behavior reported in this work is somewhat intermediate to that observed previously, possibly due to the localized loading, which can result in substantially softer microscopic behavior of the PZT with respect to its macroscopic counterpart, which is often comparable to a hard ceramic. ${ }^{[48]}$

The exhaustion of the ferroelastic variants with increasing tip pressure is also evident in the resonance frequency evolution with applied pressure (Figure 3). At increasing electric bias values, an initial small softening is observed in the curves for the lowest tip pressures, while at increasing bias and/or tip pressures, a slight hardening in the resonance loops occurs. In fact, at increasing pressure, the resonance frequency continuously increases, which is consistent with a hardening of the material and a reduction of the ferroelastic variants. We note that a tip-surface contact hardening can also be expected due to the applied tip pressure. However, the pressure exerted through the tip is constant during each piezoresponse measurement. Therefore, the increase evident in the resonance frequency during each hysteresis curve measurement can be assigned to material hardening. Such hardening of the material is also consistent with a non-reversible increase in the coercive fields with applied pressure, as seen in Figure 2a and $2 \mathrm{~b}$.

Intriguingly, when the experiments are performed on samples with a top electrode, the increasing pressure does not result in a substantial variation of the overall shape or area of the piezoresponse curves (Figure 4). The presence of a large top electrode -up to $1 \mathrm{~mm}$ in diameter, more than three orders of magnitude larger than the film thickness- results in the 


\section{WILEY-VCH}

creation of a relatively uniform electric field, regardless of the high mechanical pressure applied. The volume probed by the electric field compared to the mechanical field in the presence of a top electrode is therefore substantially larger than that in absence of a top electrode. In other words, in absence of a top electrode, both the volumes affected by the mechanical and electrical signal are comparable. Therefore, as the mechanical pressure reduces the ferroelastic variants, the electrical probing reveals clearly any eventual changes in the contributing events to the piezoresponse. However, when a top electrode is present, the volume probed by the electric field increases substantially compared to the volume affected by the mechanical field. Hence, even if a small volume of the film is affected by the applied pressure, the electrically probed response (i.e. piezoresponse hysteresis curve) is impervious to such changes. This confirms that the variations in properties induced by locally applied pressure are confined to a local region of the film. Furthermore, when no top electrode is present, the piezoresponse loops demonstrate a vertical and lateral shift under increasing applied pressure, while in the presence of an electrode, the loops remain centered. This points to a possible change in the contribution of the capacitive force in the PFM signal. Specifically, the work function of the $\mathrm{Pt}$ film on the cantilever is likely to increase under the compressive pressure resulting in a higher contact potential difference as the loading force increases. ${ }^{[49]}$ This leads to the hysteresis loops moving downwards and towards the right under increased applied pressure (Figure 2a). In contrast, the presence of a top electrode screens the electric field and thus the capacitive force between the cantilever and Pt tip is minimized.

\section{Conclusion}

In summary, stress-mediated SS-PFM was used to generate nanoscale, localized stress in a PZT film with composition in proximity of the morphotropic phase boundary, with coexisting rhombohedral and tetragonal phases. A rearrangement of the domain structure occurs through electrically- and mechanically-induced motion of ferroelastically-active domain 


\section{WILEY-VCH}

walls, suggesting strong interplay between ferroelectric and ferroelastic phenomena. Modification of hysteresis loops under increased tip pressure is consistent with an expected reduction in ferroelastic domain variants, which would in turn reduce the ferroelastic domain wall contribution to the local piezoelectric response of the film. As a result, the applied outof-plane pressure increases the in-plane polarization of the domains with respect to the out-ofplane component. This result is also validated via phase field simulations, which suggest exhaustion of ferroelastic domain variants under increasing pressure.

The stress-mediated switching spectroscopy technique discussed here is an effective tool for local probing of any electromechanical phenomena where local strain plays a significant role on the studied properties. As an example, it can be readily expanded to explore more complex electromechanical responses, such as probing piezoelectric nonlinearity as a function of applied pressure, which should allow quantification of the contribution from ferroelastic and ferroelectric domains and domain wall motion to the local piezoelectric response: a feat that even macroscopically has proved elusive in complex oxides with polymorphic structures. Furthermore, the technique can be extrapolated to other hysteretic phenomena amenable to mechanical pressure, such as ionic motion-mediated systems -with the prime example of Li-ion batteries- and more complex systems such as biological matter with both ferroelectric and ionic-motion activities -as is the case for nuclease, aminoacids etc.- where functionalities are controlled by a complex and not yet fully understood interplay of mechanical, chemical and electrical signals.

\section{Experimental Section}

Thin Film Processing. The $\mathrm{Pb}\left(\mathrm{Zr}_{0.53} \mathrm{Ti}_{0.47}\right) \mathrm{O}_{3}$ films were deposited by sol-gel spin-coating from a precursor solution with $15 \%$ excess $\mathrm{Pb}$ with respect to the stoichiometry in order to compensate for its loss at the high processing temperatures $\left(\sim 700^{\circ} \mathrm{C}\right)$. The substrates used were platinized Si wafers. The films were dense (with no obvious porosity) and highly (100)- 


\section{WILEY-VCH}

textured (>97\% Lotgering factor). Top circular electrodes were created by sputtering $80 \mathrm{~nm}$ of Pt, patterned via lift-off photolithographic approach. The full description of the processing procedure for the substrate, films and electrodes is provided elsewhere. ${ }^{[28]}$

Functional Characterization. The mechanical writing was performed by increasing the deflection set-point (i.e. the cantilever deflection voltage maintained by the atomic force microscope feedback loop proportional to the applied force or pressure) to a $\mathrm{Ti} / \mathrm{Pt}$ coated $\mathrm{Si}$ tip (Asylum Research AC240TM) while scanning. From a combination of force-distance measurements and subsequent thermal tuning methods, the spring constant of the cantilever was found to be $3.2 \mathrm{Nm}^{-1}$. Each $1 \mathrm{~V}$ of deflection setpoint from the tip corresponds to a loading force of about $200 \mathrm{nN}$.

The PFM imaging and spectroscopy were performed with $200-400 \mathrm{kHz}, 2 \mathrm{~V}$ pp band excitation signal applied to a metal-coated tip. The spatially resolved nanoscale measurements were performed on a commercial system (Cypher, Asylum Research) scanning probe microscope equipped with a Labview/Matlab based band excitation waveform generator and DAQ platform. ${ }^{[33]}$ Stress-mediated SSPFM is an adaptation of $\operatorname{SSPFM}^{[32]}$ for variable setpoints, where the set-point is varied in a triangular waveform (as illustrated in Figure S2). The tip was first brought into contact with the surface at $1 \mathrm{~V}$ deflection setpoint for all measurements. Once the contact was established, the set-point voltage was raised to a chosen value via the Labview interface. Local piezoresponse hysteresis loops were collected at this set-point before the tip pressure was increased to the next higher value. The maximum value of tip pressure was pre-defined and the pressure was subsequently reduced to the starting setpoint in a reverse step-form function. Upon the completion of data collection, the tip was moved to the next location. The spectroscopic measurements were performed at $\sim 12 \mathrm{~s} / \mathrm{pixel}$ for each chosen setpoint waveform, with $4 \mathrm{~ms}$ at each ac voltage step. Mapping of the electromechanical response was done typically on a $10 \times 10$ points grid with a spacing of 50 $\mathrm{nm}$, albeit other spacing and image sizes were also used. This gives rise to a multidimensional 


\section{WILEY-VCH}

data set of piezoresponse as a function of tip pressure, excitation voltage, and two spatial coordinates. The individual curves were fitted to a simple harmonic oscillator equation to yield the amplitude and phase at the resonance as a function of position, voltage and applied pressure, resulting in increased signal-to-noise ratio and reduced topographic crosstalk. ${ }^{[50]}$

\section{Supporting Information}

Supporting Information is available from the Wiley Online Library or from the author.

\section{Acknowledgements}

NBG gratefully acknowledges funding from the US National Science Foundation through grants \# DMR-1255379 and CMMI-1537262. A portion of this research was conducted at the Center for Nanophase Materials Sciences, which is sponsored at Oak Ridge National Laboratory by the Scientific User Facilities Division, Office of Basic Energy Sciences, U.S. Department of Energy under user proposal CNMS2013-143. The work at Penn State was supported by the U.S. Department of Energy, Office of Basic Energy Sciences, Division of Materials Sciences and Engineering under Award No. DE-FG02-07ER46417. The work was also supported by a Department of Employment and Learning Northern Ireland (DELNI) studentship.

\section{References:}

Received: ((will be filled in by the editorial staff))

Revised: ((will be filled in by the editorial staff)) Published online: ((will be filled in by the editorial staff))

[1] O. Auciello, C. A. Paz de Araujo, J. Celinska, in Emerging Non-volatile Memories (Eds. S. Hong, O. Auciello, D. Wouters) Springer, New York, pp 3-35, 2014.

[2] S. Hong, Y. Kim, in Emerging Non-volatile Memories (Eds. S. Hong, O. Auciello, D. Wouters) Springer, New York, pp 259-274, 2014. 


\section{WILEY-VCH}

[3] G. Gautschi, Piezoelectric Sensorics - Force Strain Pressure Acceleration and Acoustic Emission Sensors Materials, Springer, New York 2002.

[4] C. Daumont, W. Ren, I. C. Infante, S. Lisenkov, J. Allibe, C. Carráro, S. Fusil, E. Jacquet,

T. Bouvet, F. Bouamrane, S. Prosandeev, G. Geneste, B. Dkhil, L. Bellaiche, A. Barthámy, M. Bibes, J. Phys. Condens. Matter 2012, 24, 162202.

[5] J. S. Speck, W. Pompe, J. Appl. Phys. 1994, 76, 466.

[6] K. Lefki, G. J. M. Dormans, J. Appl. Phys. 1994, 76, 1764.

[7] K. J. Choi, M. Biegalski, Y. L. Li, A. Sharan, J. Schubert, R. Uecker, P. Reiche, Y. B.

Chen, X. Q. Pan, V. Gopalan, L.-Q. Chen, D. G. Schlom, C. B. Eom, Science 2004, 306, 1005.

[8] J. H. Haeni, P. Irvin, W. Chang, R. Uecker, P. Reiche, Y. L. Li, S. Choudhury, W. Tian, M. E. Hawley, B. Craigo, A. K. Tagantsev, X. Q. Pan, S. K. Streiffer, L. Q. Chen, S. W. Kirchoefer, J. Levy, D. G. Schlom, Nature 2004, 430, 758.

[9] J. Dvorak, Y. U. Idzerda, S. B. Ogale, S. Shinde, T. Wu, T. Venkatesan, R. Godfrey, R. Ramesh, J. Appl. Phys. 2005, 97, $10 \mathrm{C} 102$.

[10] E. Koray Akdogan, W. K. Simon, A. Safari, IEEE Trans. Ultrason. Ferroelectr. Freq. Control. 2006, 53, 2323.

[11] A. Y. Emelyanov, N. A. Pertsev, A. L. Kholkin, Phys. Rev. B 2002, 66, 214108.

[12] B. Jaffe, W. R. Cook, H. Jaffe, Piezoelectric Ceramics, Academic Press, London 1971.

[13] J. Y. Li, R. C. Rogan, E. Ustundag, K. Bhattacharya, Nat. Mater. 2005, 4, 776. 


\section{WILEY-VCH}

[14] A. Pramanick, A. D. Prewitt, J. S. Forrester, J. L. Jones, Crit. Rev. Solid State Mater. Sci. 2012, 37, 243.

[15] D. M. Marincel, H. R. Zhang, J. Britson, A. Belianinov, S. Jesse, S. V. Kalinin, L. Q.

Chen, W. M. Rainforth, I. M. Reaney, C. A. Randall, S. Trolier-McKinstry, Phys. Rev. B 2015, 91, 134113.

[16] H. Xu, D. Lee, S. B. Sinnott, V. Dierolf, V. Gopalan, S. R. Phillpot, J. Phys. Condens. Matter 2010, 22, 135002.

[17] F. Griggio, S. Jesse, A. Kumar, O. Ovchinnikov, H. Kim, T. N. Jackson, D. Damjanovic, S. V. Kalinin, S. Trolier-McKinstry, Phys. Rev. Lett. 2012, 108, 157604.

[18] P. Bintachitt, S. Jesse, D. Damjanovic, Y. Han, I. M. Reaney, S. Trolier-McKinstry, S. V. Kalinin, Proc. Natl. Acad. Sci. U.S.A. 2010, 107, 7219.

[19] J. Woo, S. Hong, D. K. Ming, H. Shin, K. S. No, Appl. Phys. Lett. 2002, 80, 4000

[20] S. Jesse, B. J. Rodriguez, S. Choudhury, A. P. Baddorf, I. Vrejoiu, D. Hesse, M. Alexe, E. A. Eliseev, A. N. Morozovska, J. Zhang, L. Chen, S. V. Kalinin, Nat. Mater. 2008, 7, 209.

[21] D. M. Marincel, H. Zhang, A. Kumar, S. Jesse, S. V. Kalinin, W. M. Rainforth, I. M. Reaney, C. A. Randall, S. Trolier-McKinstry, Adv. Funct. Mater. 2014, 24, 1409.

[22] B. D. Huey, C. Ramanujan, M. Bobji, J. Blendell, G. White, R. Szoszkiewicz, A. Kulik, J. Electroceram. 2004, 13, 287.

[23] R. Xu, S. Liu, I. Grinberg, J. Karthik, A. R. Damodaran, A. M. Rappe, L. W. Martin, Nat. Mat. 2015, 14, 79.

[24] V. Koval, M. J. Reece, A. J. Bushby, J. Appl. Phys. 2005, 97, 07401. 


\section{WILEY-VCH}

[25] H. Nili, K. Kalantar-zadeh, M. Bhaskaran, S. Sriram, Prog. Mater. Sci. 2013, 58, 1.

[26] G. A. Schneider, T. Scholz, J. Munoz-Saldana, M. V. Swain, Appl. Phys. Lett. 2005, 86, 192903.

[27] H. Lu, C.-W. Bark, D. E. Ojos, J. Alcala, C. B. Eom, G. Catalan, A. Gruverman, Science 2012, 336, 59.

[28] Y. Bastani, T. Schmitz-Kempen, A. Roelofs, N. Bassiri-Gharb, J. Appl. Phys. 2011, 109, 014115.

[29] Y. Heo, B. Jang, S. J. Kim, C. Yang, J. Seidel, Adv. Mater. 2014, 26, 7568.

[30] Y. Kim, S. Bühlmann, S. Hong, S. H. Kim, K. No, Appl. Phys. Lett. 2007, 90, 072910.

[31] Y. Choi, P. Sharma. C. Phatak, D. J. Gosztola, Y. Liu, J. Lee, B. Lee, J. Li, A. Gruverman, S Ducharme, S. Hong, ACS Nano 2015, 9, 1809.

[32] R. K. Vasudevan, S. Jesse, Y. Kim, A. Kumar, S. V. Kalinin, MRS Commun. 2012, 2, 61.

[33] S. Jesse, S. V. Kalinin, R. Proksch, A. P. Baddorf, B. J. Rodriguez, Nanotechnology 2007, 18, 435503.

[34] S. Guo, O. S. Ovchinnikov, M. E. Curtis, M. B. Johnson, S. Jesse, S. V. Kalinin, J. Appl. Phys. 2010, 108, 084103.

[35] L.-Q Chen, J. Am. Ceram. Soc. 2008, 91, 1835.

[36] Y. L. Li, S. Y. Hu, Z. K. Liu, L. Q. Chen, Acta Materialia 2002, 50, 395.

[37] A. Fischer-Cripps, Introduction to Contact Mechanics, Spinger Verlag, London 2000. 


\section{WILEY-VCH}

[38] Y. Kim, S. J. Kelly, A. Morozovska, E. K. Rahani, E. Strelcov, E. Eliseev, S. Jesse, M.

D. Biegalski, N. Balke, N. Benedek, D. Strukov, J. Aarts, I. Hwang, S. Oh, J. S. Choi, T. Choi, B. H. Park, V. B. Shenoy, P. Maksymovych, S. V. Kalinin, Nano Lett. 2013, 13, 4068.

[39] A. K. Tagantsev, P.Muralt and J. Fousek, J. Mat. Res. Soc. Symp. Proc. 2004, 784, C10.6.1.

[40] I. M. Reaney, D. V. Taylor, K. G. Brooks, J. Sol-Gel Sci. Technol. 1998, 13, 813.

[41] A. Wu, P. M. Vilarinho, V. V. Shvartsman, G. Suchaneck, A. L. Kholkin, Nanotechnology 2005, 16, 2587.

[42] H. J. Kim, S. H. Oh, H. M. Jang, Appl. Phys. Lett. 1999, 75, 3195.

[43] M. Park, S. Hong, J. Kim, J. Hong, K. No, Appl. Phys. Lett. 2011, 99, 142909.

[44] N. Bassiri-Gharb, S. Trolier-McKinstry, D. Damjanovic, J. Appl. Phys. 2006, 100, 044107.

[45] N. Bassiri-Gharb, S. Trolier-McKinstry, D. Damjanovic, J. Appl. Phys. 2011, 110, 124104.

[46] G. Zavala, J. Fendler, S. Trolier-McKinstry, J. Appl. Phys. 1997, 81, 7480.

[47] H. H. A. Krueger, J. Acoust. Soc. Am. 1967, 42, 636.

[48] F. Xu, S. Trolier-McKinstry, W. Ren, B. Xu, Z,-L Xie, K. J. Hemker, J. Appl. Phys. 2001, 89, 1336.

[49] S. Hong, J. Woo, H. Shin, J. U, Jeon, Y. E. Pak, E. L. Colla, N. Setter, E. Kim, K. No, J. Appl. Phys. 2001, 89, 1377. 


\section{WILEY-VCH}

[50] S. Jesse, S. Guo, A. Kumar, B. J. Rodriguez, R. Proksch, S. V. Kalinin, Nanotechnology

2010, 21, 405703.

\section{FIGURES}

a

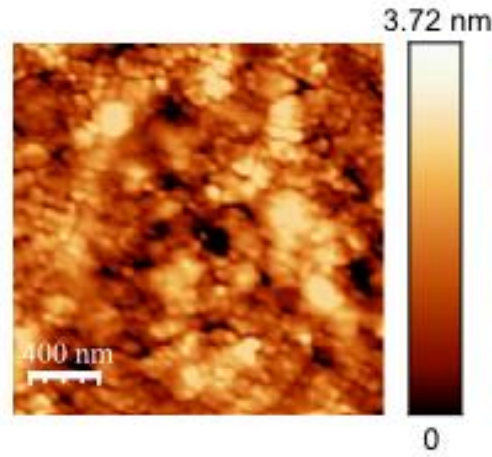

d

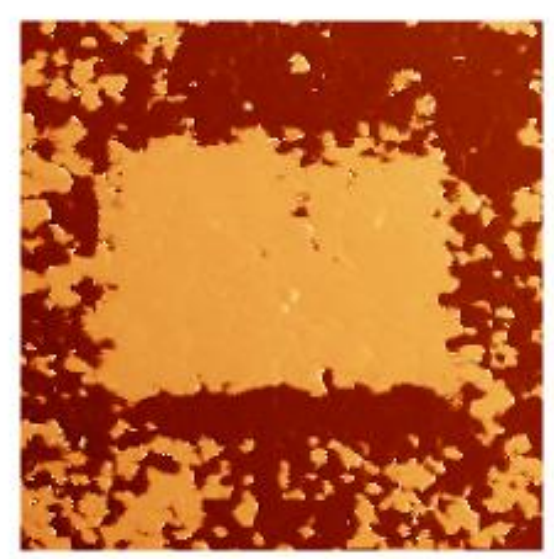

b

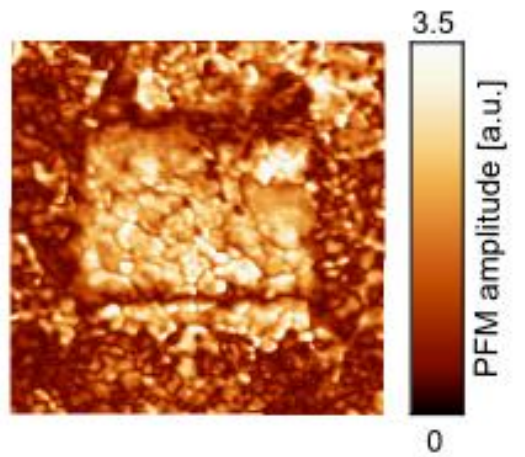

C

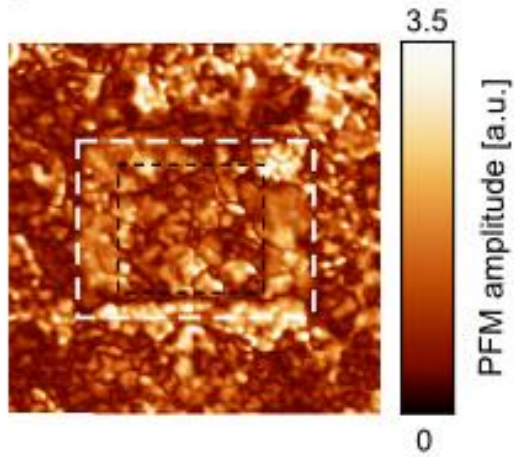

$200 \quad$ e

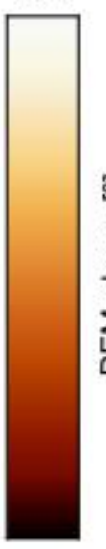

$-160$

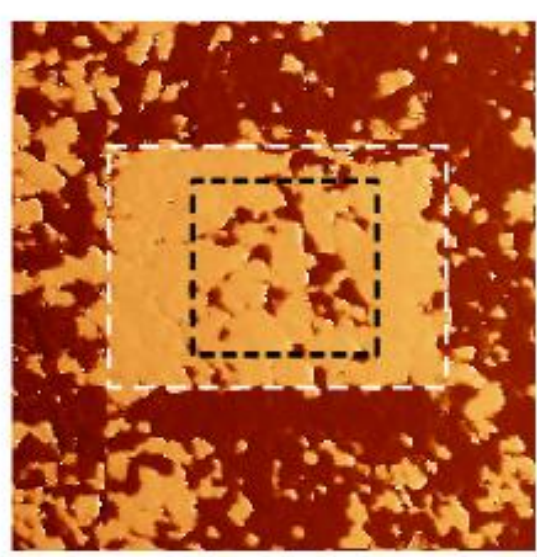

200

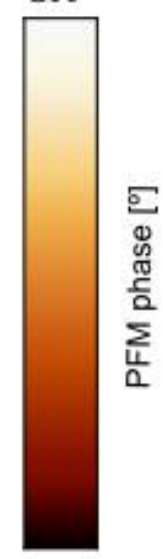

$-160$

Figure 1. Pressure induced writing on a PZT film. (a) Topography, PFM (b) amplitude and (d) phase map of a central region written with $-6 \mathrm{~V}$ DC bias applied through the tip. PFM (c) amplitude and (e) phase map of the same region (bias written), after a loading force of 1000 $\mathrm{nN}$ was applied from the tip to the smaller region indicated by the black dashed lines. A comparison of $(b, d)$ with $(c, e)$ shows reorganization of the domain structure upon application of loading force through the PFM tip. 


\section{WILEY-VCH}

a

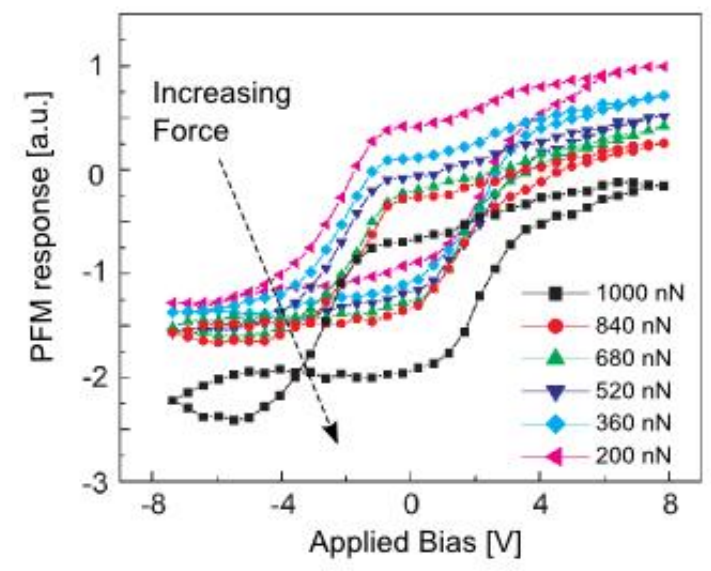

C

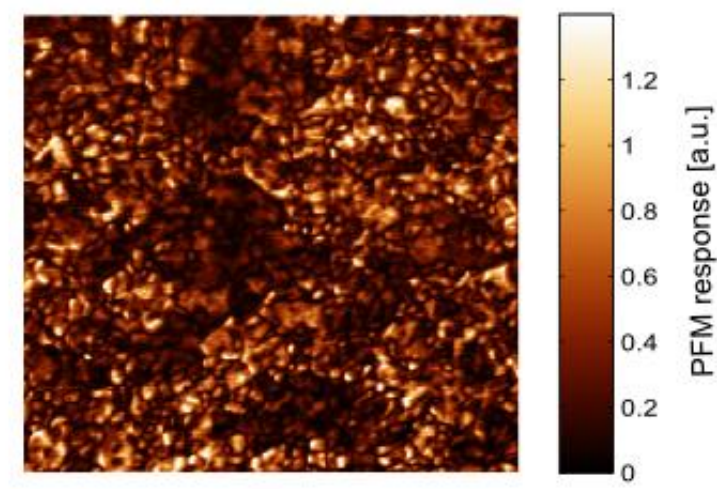

b

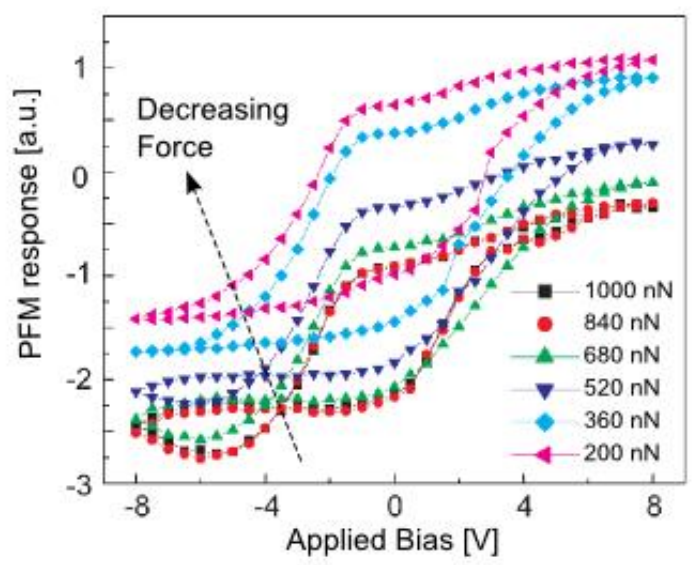

d

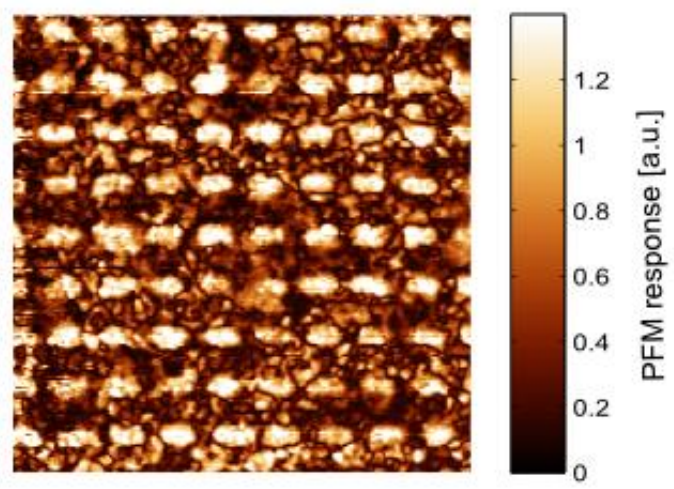

Figure 2. Piezoresponse hysteresis loops collected on a PZT film with no top electrode. Piezoresponse curves as a function of (a) increasing and (b) decreasing tip force. PFM amplitude map of the region on the film (c) before the loops were collected, and (d) after the piezoresponse data were collected. 
a

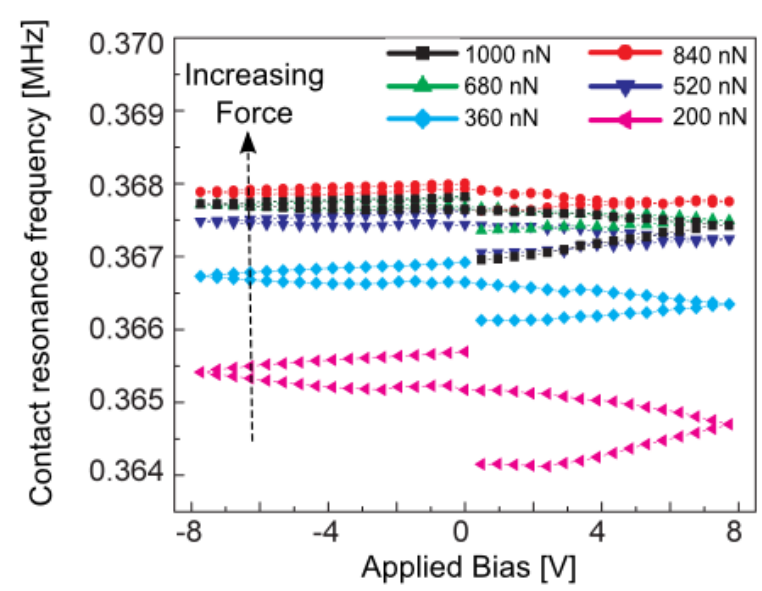

b

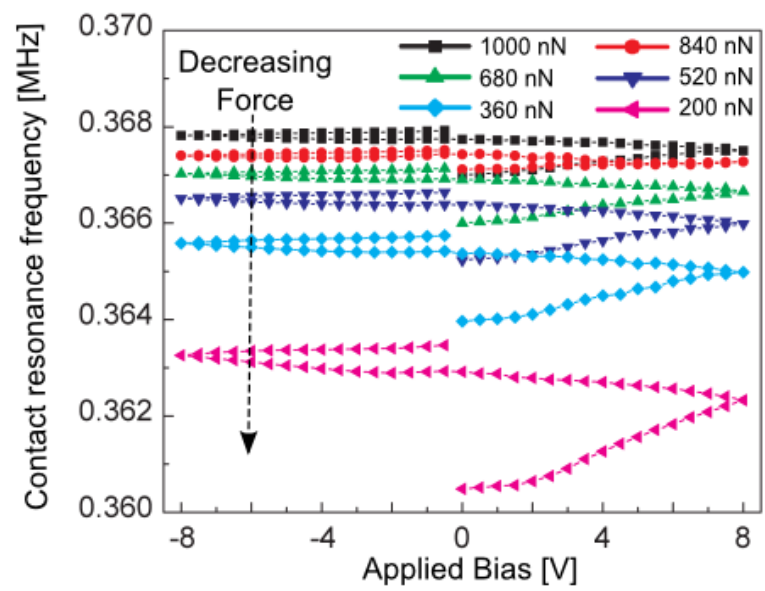

Figure 3. Variation of contact resonance frequency as a function of applied bias and loading force. The resonance frequency is shown within each hysteresis data collection, with (a) increase in tip force and (b) decrease in tip force.

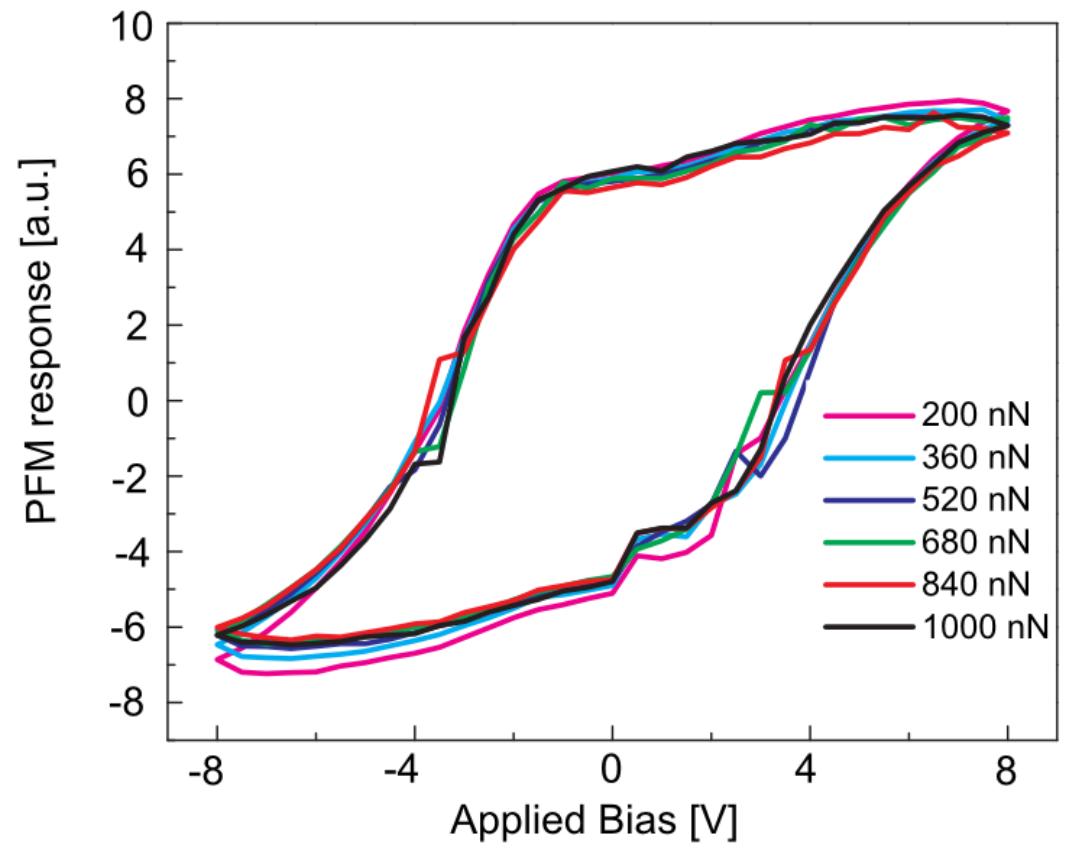

Figure 4. Piezoresponse loops collected on a PZT film with a top electrode, as a function of increasing loading force. 


\section{WILEY-VCH}

a

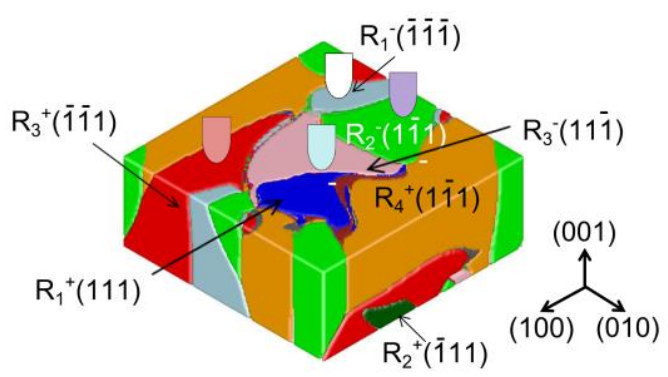

b

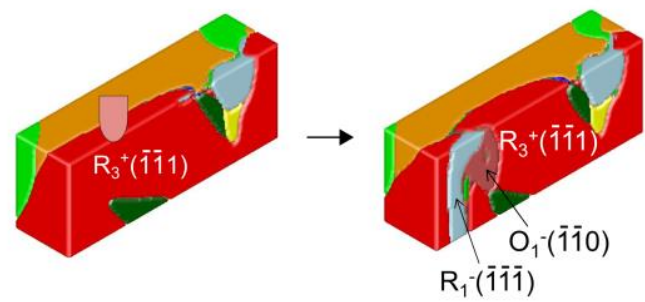

C

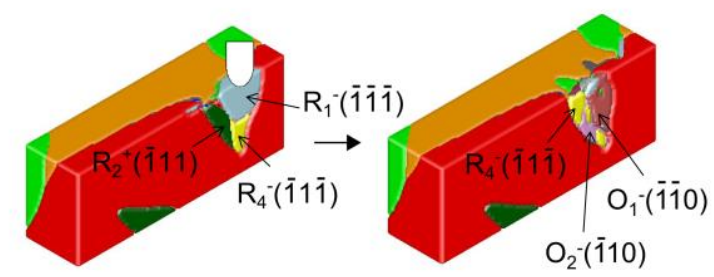

d

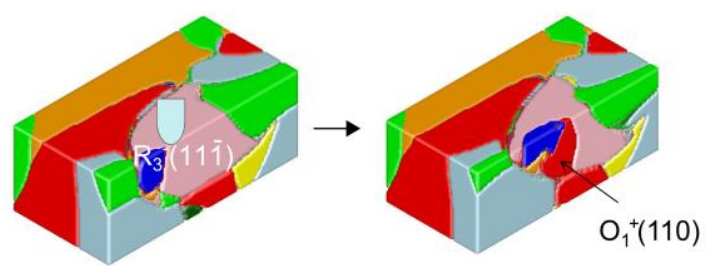

e

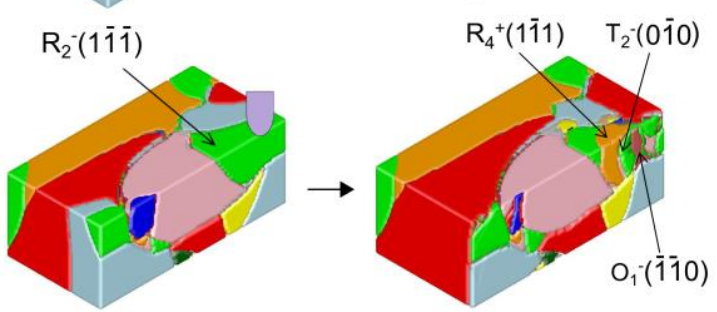

Figure 5. Phase field modeling simulations of domain structure in a PZT film in presence of local mechanical loading. (a) Multi-domain structure of $\mathrm{Pb}\left(\mathrm{Zr}_{1-\mathrm{x}} \mathrm{Ti}_{\mathrm{x}}\right) \mathrm{O}_{3}$ at $\mathrm{x}=0.47$ in equilibrium state. Domain structures before and after mechanical switching when tip is brought into contact with the sample (b) entirely in the $\mathrm{R}_{3}{ }^{+}(\overline{1} \overline{1} 1)$ domain, (c) in the entire $\mathrm{R}_{1}{ }^{-}$ ( $\overline{1} \overline{1} \overline{1})$ domain, (d) in the entire $\mathrm{R}_{3}{ }^{-}(11 \overline{1})$ domain, (e) in the entire $\mathrm{R}_{2}{ }^{-}(1 \overline{1} \overline{1})$ domain. 

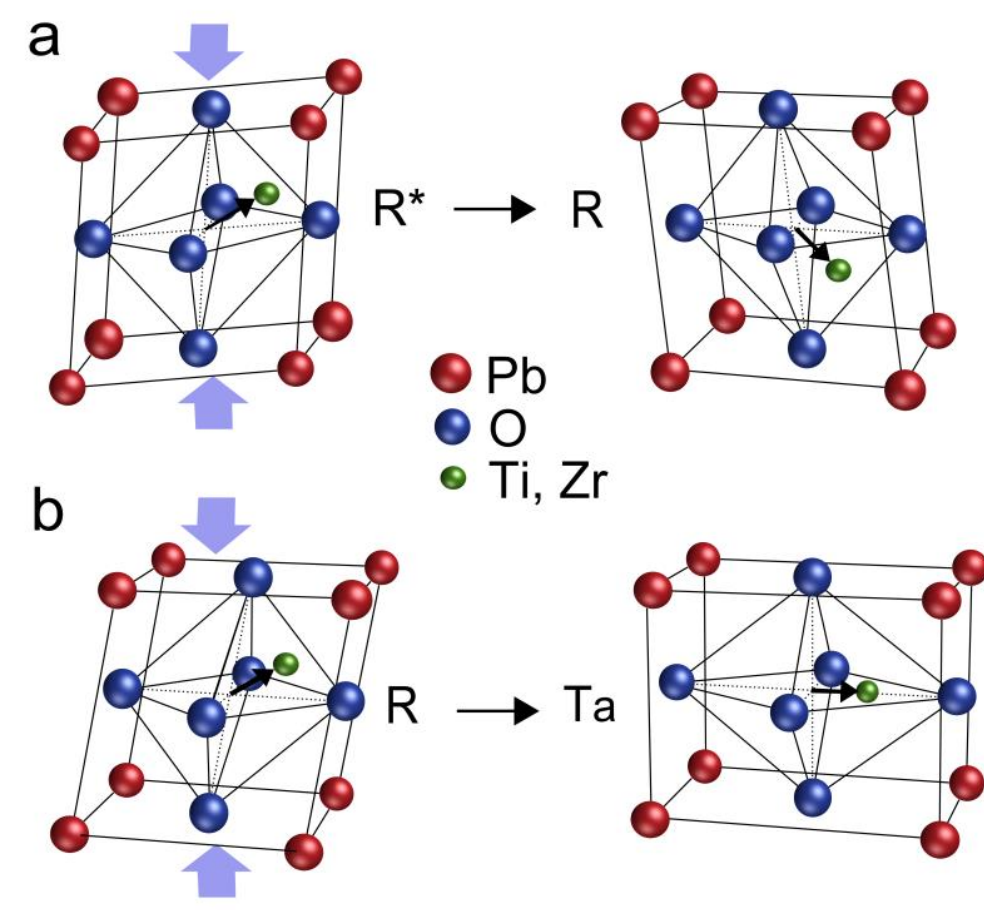

- $\mathrm{Ti}, \mathrm{Zr}$
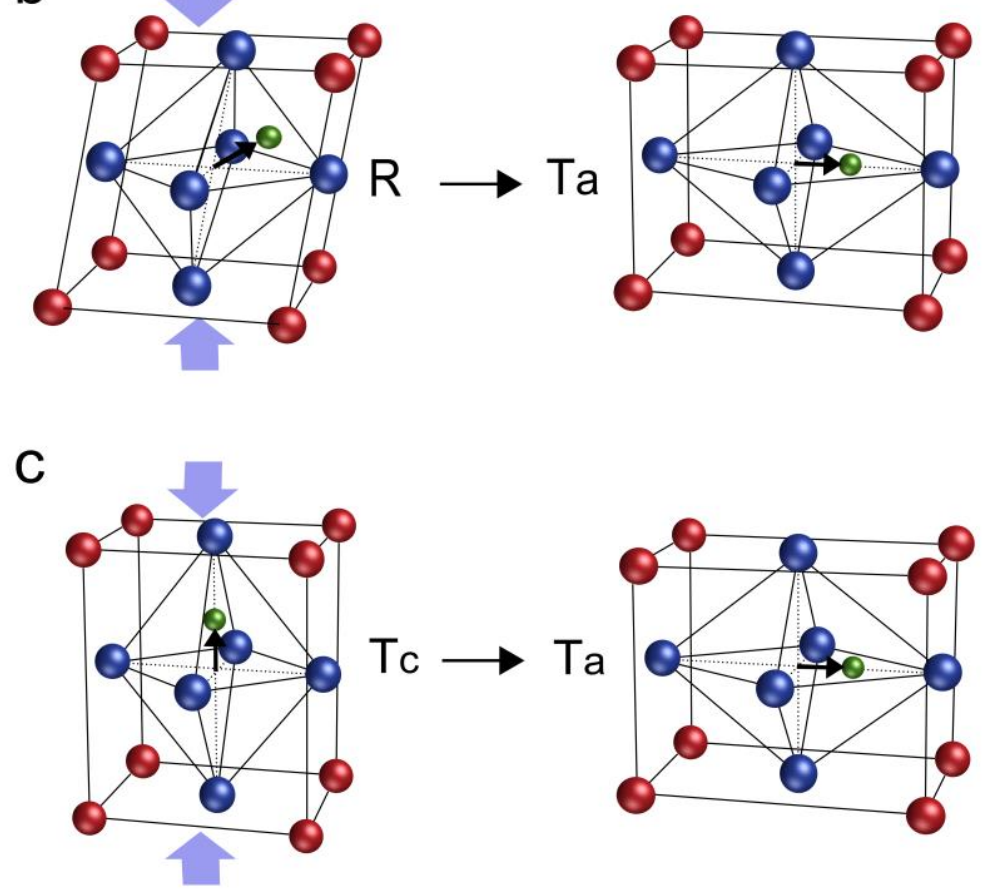

Figure 6. Schematic representation of polarization re-orientation and accompanying change in perovskite crystal deformation as a result of axial loading. (a) Polarization reorientation in a "misoriented" rhombohedral cell with non-degenerate polarization directions $\left(\mathrm{R}^{*} \rightarrow \mathrm{R}\right)$. (b) Pressure induced rhombohedral to tetragonal " $a$ " $\left(\mathrm{R} \rightarrow \mathrm{T}_{\mathrm{a}}\right)$ and (c) tetragonal " $c$ " to " $a$ " ( $\mathrm{T}_{\mathrm{c}}$ $\rightarrow \mathrm{T}_{\mathrm{a}}$ ) switching. The atomics displacements are exaggerated to highlight the resulting polarization direction and crystal deformation. 


\section{WILEY-VCH}

The effect of applied mechanical pressure on the local hysteretic response on a $\mathrm{Pb}\left(\mathrm{Zr}_{0.53} \mathrm{Ti}_{0.47}\right) \mathrm{O}_{3}$ film is studied through a novel scanning probe microscopy based technique. The modified shape of the piezoresponse hysteresis curves, change in resonance frequency, as well as phase field modeling provide a coherent and direct insight into local ferroelastic and ferroelectric switching events in the films.

\section{Ferroics}

David Edwards, Steven Brewer, Ye Cao, Stephen Jesse, Long-Qing Chen, Sergei V. Kalinin, Amit Kumar* and Nazanin Bassiri-Gharb*

Local probing of ferroelectric and ferroelastic switching through stress-mediated piezoelectric spectroscopy
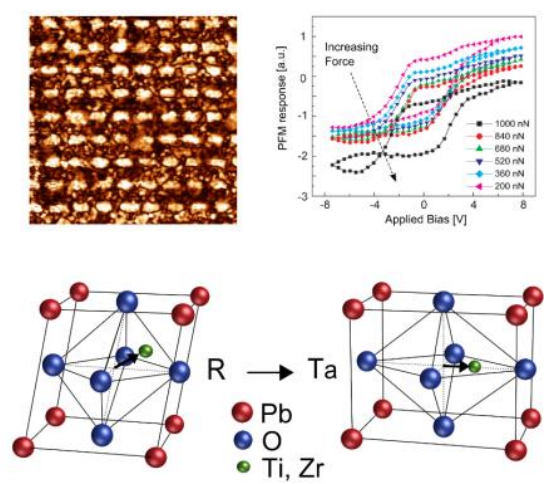